\title{
Electrical TCAD Study of the Low-Voltage Avalanche-Mode Superjunction LED
}

\author{
R.J.E. Hueting, Senior Member, IEEE, H. de Vries, S. Dutta, and A.J. Annema, Member, IEEE
}

\begin{abstract}
The CMOS silicon avalanche-mode lightemitting diode (AMLED) has emerged as a potential light source for monolithic optical interconnects. Earlier we presented a superjunction light-emitting diode (SJLED) that offers a higher electroluminescent intensity compared to a conventional AMLED because of its more uniform field distribution. However, for reducing power consumption lowvoltage ( $\leq 15 \mathrm{~V})$ SJLEDs are desired, not explored before. In this work we present a TCAD simulation feasibility study of the low-voltage SJLED for various doping concentrations and device dimensions. The results show that for obtaining a constant field, approximately a tenfold more aggressive charge balance condition in the SJLED is estimated than traditionally reported. This is important for establishing a guideline to realize optimized RESURF and SJLEDs in the ever-shrinking advanced CMOS nodes.
\end{abstract}

Index Terms-Avalanche breakdown, Diode, LightEmitting Diode (LED), Power, silicon

\section{INTRODUCTION}

In the 1950's it was discovered that silicon (Si) pnjunctions operating in avalanche breakdown exhibit broadspectrum electro-luminescence (EL) at short wave lengths ( $\lambda \sim 350-900 \mathrm{~nm})$, although with a low internal quantum efficiency $\left(\eta_{\mathrm{RAD}} \sim 10^{-5}\right)$ [1], [2]. After this discovery it took practically half a century for research on $\mathrm{Si}$ avalanche-mode light emitting diodes (AMLEDs) to gain momentum ( [3]- [9]). This can be partly attributed to the advancement of commercial CMOS technology driven by the strong demand for more onchip functionality. In addition, Si AMLEDs exhibit significant spectral overlap with the responsitivity of $\mathrm{Si}$ photodiodes [10] which is beneficial for on-chip optical interconnects.

Due to a wide variety of commercial CMOS technologies, various approaches have been reported to increase $\eta_{\mathrm{RAD}}$ of AMLEDs:

- Additional carrier injection via a third terminal in AMLEDs [11].

- Carrier energy and momentum engineering [12], [13].

- The superjunction light-emitting diode (SJLED) [14], [15].

In this work we focus on the last approach. The basic idea of the SJLED is to mimic a p-i-n diode, see Fig. 1(a), at avalanche breakdown. The constant electric field distribution

R.J.E. Hueting, H. de Vries, and A.J. Annema are with the University of Twente, 7500 AE Enschede, The Netherlands.

S. Dutta is with Delft University of Technology, 2600 AA Delft, The Netherlands.

Corresponding author: R.J.E. Hueting, University of Twente, 7500 AE Enschede, The Netherlands (email: r.j.e.hueting@utwente.nl)
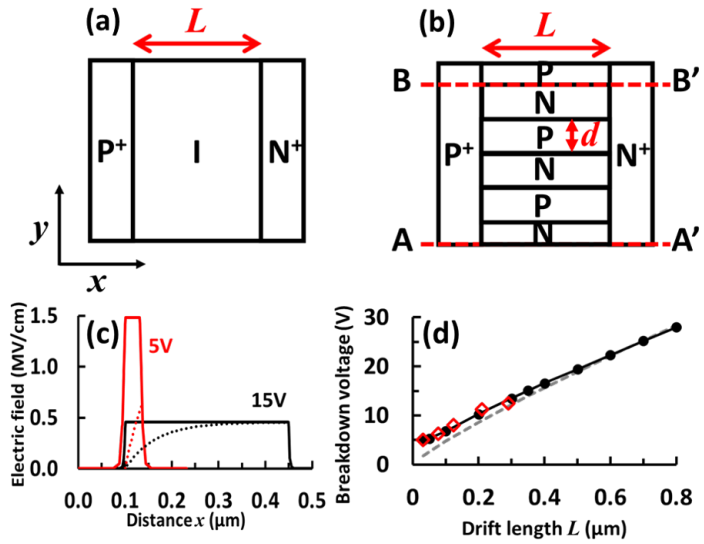

Fig. 1. Schematic top view of the (a) p-i-n diode and (b) SJLED. In theory the $p / n$ poles are infinitely repeated in the $y$-direction. Line $A$ $A^{\prime}$ indicates the axis of symmetry of the device. The drift length of both devices and pole width are indicated by $L$ respectively $d$. The anode/cathode regions have a doping of $10^{19} \mathrm{~cm}^{-3}$. (c) Breakdown field distributions of a $5 \mathrm{~V}$ and $15 \mathrm{~V} \mathrm{p}$-i-n diode compared to their effective field obtained from a 1D nonlocal avalanche model (dotted curves) using Eqs. (3)-(4). (d) Breakdown voltage $(\boldsymbol{B} \boldsymbol{V})$ against $\boldsymbol{L}$ of the Si p-i-n diode obtained from TCAD simulations [23] and measurements (open red symbols) [27]. The grey dashed line represents results obtained from Fulop's approximation [30], showing a discrepancy for smaller $\boldsymbol{L}$.

at breakdown $\left(\mathcal{E}_{x}(x)\right)$ in the drift (or "active") region of the p-i-n diode, see Fig. 1(c), results in a higher EL-intensity thus $\eta_{\mathrm{RAD}}$ compared to conventional pn junctions with the same breakdown voltage $(B V)$ [14]. In the latter $\mathcal{E}_{x}(x)$ is triangularly shaped and hence only near the peak field, light emission spots will form.

However, to realize a p-i-n diode in standard CMOS technology is difficult. Therefore, the widely adopted reduced surface field (RESURF) effect [16], [17], [18] in power devices is used by placing multiple parallel p/n-layers or "poles", i.e. superjunction RESURF, see Fig. 1(b). In this way the p/n poles can be fully depleted at avalanche breakdown akin to the intrinsic region of a $\mathrm{p}-\mathrm{i}-\mathrm{n}$ diode.

For obtaining the optimal RESURF condition, thus a constant $\mathcal{E}_{x}(x)$, the product of pole width $(d)$ and pole doping concentration $(N)$ must satisfy the charge balance condition [19], [17], [16]:

$$
N \cdot d \lesssim\left|\mathcal{E}_{\mathrm{c}, y}\right| \frac{2 \varepsilon_{\mathrm{s}}}{q},
$$

where $\mathcal{E}_{\mathrm{c}, y}$ is the critical (or breakdown) field of the one dimensional (1D) vertical ( $y$-direction) $\mathrm{p} / \mathrm{n}$ poles, $\varepsilon_{\mathrm{s}}$ is the permittivity, and $q$ is the elementary charge. Typically, the charge balance condition in $\mathrm{Si}$ is reported to be $N \cdot d \lesssim 10^{12}$ 
$\mathrm{cm}^{-2}$ [16], but this value increases for higher $N\left(\gtrsim 10^{18}\right.$ $\mathrm{cm}^{-3}$ ) since the ionization rate strongly depends on the field; this increases $\mathcal{E}_{\mathrm{c}, y}$ of the $1 \mathrm{D} \mathrm{p} / \mathrm{n}$ poles [20]. Moreover because of mobility reduction $\mathcal{E}_{\mathrm{c}, y}$ increases with $N$ as well. Provided that Eq. (1) holds, the $B V$ of the SJLED is determined by $\mathcal{E}_{\mathrm{c}, x}$, as in the p-i-n diode.

At breakdown the constant field in the p-i-n diode (and optimized SJLED) reaches the critical field $\left(\mathcal{E}_{x}(x)=\mathcal{E}_{\mathrm{c}, x}\right)$ uniformly. Consequently, as shown in Fig. 1 (d) $B V \approx\left|\mathcal{E}_{\mathrm{c}, x}\right|$. $L$, where $L$ is the drift length. Importantly, for low-voltage $(\mathrm{LV})$ devices $\mathcal{E}_{\mathrm{c}, x}$ strongly increases for smaller $L$, see also Fig. 1(c). It can be derived that

$$
\left|\mathcal{E}_{\mathrm{c}, x}\right|=\frac{b_{n}}{\ln \left(a_{n} L\right)},
$$

where $a_{n}=7.03 \cdot 10^{5} \mathrm{~cm}^{-1}$ and $b_{n}=1.23 \cdot 10^{6} \mathrm{~V} / \mathrm{cm}$ for Si.

Particularly for reducing power consumption, LV SJLEDs are desired but for $B V<5 \mathrm{~V}$ nonlocal avalanche (NLA) effects will dramatically drop the EL-intensity [21], obviously not desired. In addition, band-to-band tunneling (BTBT) effects [22] will then play a role. So far SJLEDs have been studied for $B V \gtrsim 25 \mathrm{~V}$. In this work we report a TCAD simulation study to investigate the impact of the $N \cdot d$ value on the uniformity of $\mathcal{E}_{x}(x)$ and BTBT effects in LV SJLEDs ( $B V \leq 15 \mathrm{~V}$ ), both important for increasing $\eta_{\mathrm{RAD}}$, aiming at (relatively) LV light generation.

\section{Results And discussion}

Earlier, we reported TCAD and experimental data of SJLEDs for $25 \mathrm{~V} \leq B V \leq 50 \mathrm{~V}$ [14]. Best results were obtained for $N \approx 2 \cdot 10^{17} \mathrm{~cm}^{-3}$ and a minimum $d \approx 0.38 \mu \mathrm{m}$, both defined by technology constraints. The SJLEDs $(L=2$ $\mu \mathrm{m})$ showed about a 1.7 fold increase in breakdown voltage $(B V=50 \mathrm{~V})$ compared to that of conventional (pn-junction) AMLEDs $(B V=29 V)$ of the same size and realized in the same technology. Also, the EL intensity that was measured for the same current from $400 \mathrm{~nm}$ to $870 \mathrm{~nm}$ (with a peak at $650 \mathrm{~nm}$ ) wavelength, was almost 2 times higher than that of conventional counterparts as confirmed by the two times higher measured coupling efficiency $\left(\sim 8 \cdot 10^{-9}\right)$ for the former. Those reported results therefore show that the SJLED has a more uniform field distribution. However for that SJLED, $N \cdot d \approx 7.7 \cdot 10^{12} \mathrm{~cm}^{-2}$ which is higher than the charge balance condition [16], implying that so far we did not obtain optimal RESURF.

Fig. 2 shows $\mathcal{E}_{x}(x)=\mathcal{E}_{\mathrm{c}, x}$ of the $15 \mathrm{~V}$ p-i-n diode $(L=$ $350 \mathrm{~nm}$ ) obtained from TCAD simulations [23]. Throughout this work we have used Selberherr's local impact ionization model [24] that considers for fields higher than $4 \cdot 10^{5} \mathrm{~V} / \mathrm{cm}$ (applicable in this work) impact ionization coefficients according to $\alpha_{n(p)}=a_{n(p)} \cdot \exp \left(-\left(\frac{b_{n(p)}}{\mathcal{E}}\right)^{\beta_{n(p)}}\right)$, where $a_{p}$ $=6.71 \cdot 10^{5} \mathrm{~cm}^{-1}, b_{p}=1.69 \cdot 10^{6} \mathrm{~V} / \mathrm{cm}$, and $\beta_{n(p)}=1$. For comparison, $\mathcal{E}_{x}(x)$ is shown of the SJLED with $N \cdot d \approx$ $7.7 \cdot 10^{12} \mathrm{~cm}^{-2}$ [14], taken from the line A-A', see Fig. 1(b). As expected, in the SJLED $\mathcal{E}_{x}(x)$ is not constant: at the left junction there is a peak field. This will result in more light emission near this junction (this could not be observed in our

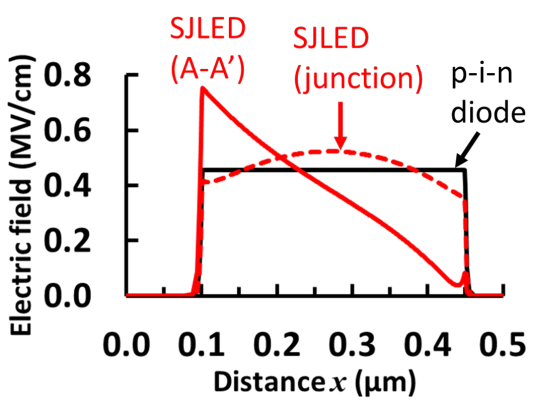

Fig. 2. Breakdown field distribution of the $15 \mathrm{~V} \mathrm{p}$-i-n diode and $15 \mathrm{~V}$ SJLED obtained from TCAD simulations (both $L=350 \mathrm{~nm}$ ), using reported values for $N \approx 2 \cdot \mathbf{1 0}^{\mathbf{1 7}} \mathrm{cm}^{-3}$ and $\boldsymbol{d} \approx 0.38 \mu \mathrm{m}$ [14]. For the SJLED $\mathcal{E}_{x}(x)$ is taken from line A-A' (Fig. 1(b)), and through the junction of a p/n pole as indicated by, e.g., line B-B' (Fig. 1(b)).

experiments [14] possibly due to the limited image resolution) rather than throughout the whole drift region as would be the case for the p-i-n diode. Clearly, for increasing the EL intensity the latter is desired.

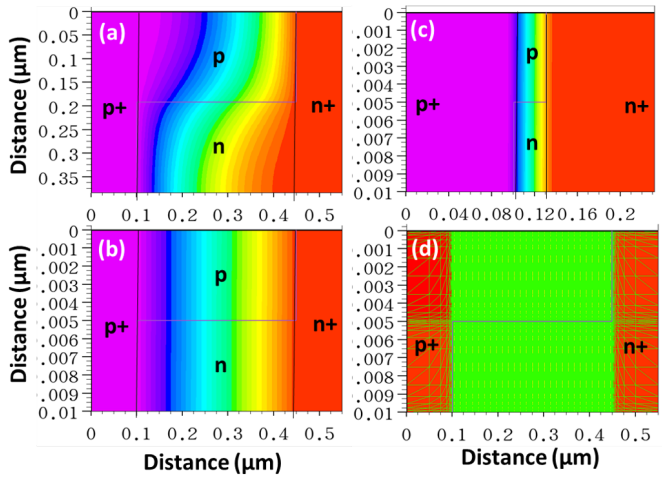

Fig. 3. 2D potential contour plots at breakdown in unit cells of (a) the SJLED for $N \approx 2 \cdot 10^{17} \mathrm{~cm}^{-3}, d \approx 0.38 \mu \mathrm{m}, L=350 \mathrm{~nm}$ (see also Fig. 2), (b) and (c) the optimized SJLED for $N=10^{18} \mathrm{~cm}^{-3}$ and $\boldsymbol{d}=$ $10 \mathrm{~nm}$ and $\boldsymbol{L}=350 \mathrm{~nm}$, respectively, $32 \mathrm{~nm}$. (d) Example of a 2D meshing plot used for obtaining figure (b) showing a dense mesh in the drift region (350 mesh points in $\boldsymbol{x}$-direction and 30 mesh points in $\boldsymbol{y}$-direction).

We have performed extensive TCAD simulations to optimize the SJLED for $B V=5 \mathrm{~V}$ and $15 \mathrm{~V}$ by incorporating three doping concentrations: $N=10^{16}, 2 \cdot 10^{17}$, and $10^{18} \mathrm{~cm}^{-3}$.

Fig. 3(a) depicts a 2D potential contour plot at breakdown in a unit cell of the SJLED with previously mentioned values of $N$ and $d$ [14], showing that the potential lines are not equidistant and strongly curved. For comparison contour plots are shown of optimized counterparts for $N=10^{18} \mathrm{~cm}^{-3}, d=10 \mathrm{~nm}$ and $L=350 \mathrm{~nm}$ (Fig 3(b)), respectively, 32nm (Fig 3(c)) both indicating optimal RESURF. For the p-i-n diode the same results are obtained where we have used about $1,200(4,000)$ mesh points for $L=32 \mathrm{~nm}(350 \mathrm{~nm})$, in which the drift region has 32 (350) mesh points in $x$-direction and 10 mesh points in $y$-direction. For the optimization of the SJLED we have used about 1,200-22,000 mesh points depending the doping and size. Here, in the drift region we have used for $L=32 \mathrm{~nm}$ (350nm) 32 (350) mesh points in $x$-direction and 30-40 mesh points in $y$-direction, see for example Fig. 3(d).

The optimization routine we have used is as follows. First $L$ needs to be determined for the $5 \mathrm{~V}$ and $15 \mathrm{~V}$ p-i-n diodes, 


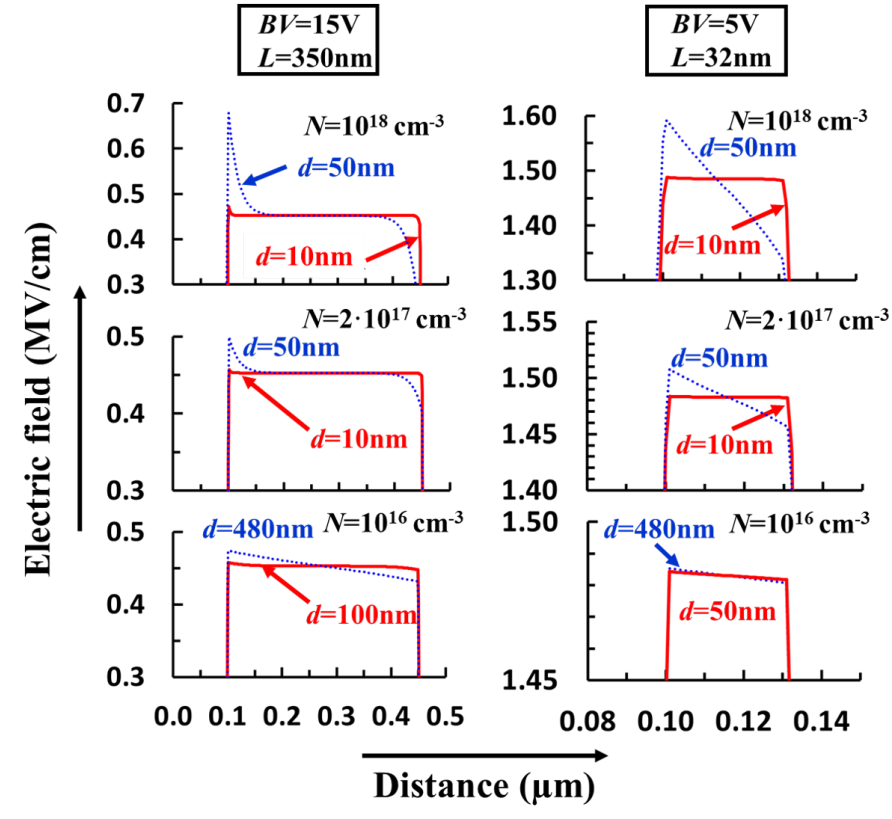

Fig. 4. Lateral breakdown field distribution obtained from TCAD simulations of $15 \mathrm{~V}$ (left) and 5V SJLEDs for $N=10^{18} \mathrm{~cm}^{-3}$ (top), $2 \cdot 10^{17} \mathrm{~cm}^{-3}$ (middle) and $10^{16} \mathrm{~cm}^{-3}$ (bottom). In all cases $d$ is varied for obtaining a constant field for $\boldsymbol{d}=100 \mathrm{~nm}\left(N=10^{16} \mathrm{~cm}^{-3}, 15 \mathrm{~V}\right)$ and $\boldsymbol{d}=10 \mathrm{~nm}\left(\boldsymbol{N}=2 \cdot 10^{17}\right.$ and $\left.10^{18} \mathrm{~cm}^{-3}\right)$. One exception: for $5 \mathrm{~V}$ and $N$ $=10^{16} \mathrm{~cm}^{-3} \boldsymbol{N} \cdot \boldsymbol{d}$ has hardly any effect.

see Fig. 1(d). These lengths, 32nm resp. 350nm long, are then used for the $5 \mathrm{~V}$ respectively $15 \mathrm{~V}$ SJLEDs. Next, for each $L$ $d$ is varied for all $N$ such that a (practically) constant $\mathcal{E}_{x}(x)$ is obtained. Fig. 4 summarizes $\mathcal{E}_{x}(x)$ for all SJLEDs. Using Eq. (1) from this part of the study it can be concluded that $N \cdot d \lesssim 10^{11} \mathrm{~cm}^{-2}$ for $N=10^{16}$ and $2 \cdot 10^{17} \mathrm{~cm}^{-3}$, while $N \cdot d \lesssim 10^{12} \mathrm{~cm}^{-2}$ for $N=10^{18} \mathrm{~cm}^{-3}$. For the latter $N \cdot d$ is estimated higher because of the higher $\mathcal{E}_{\mathrm{c}, y}(\sim 1.2 \mathrm{MV} / \mathrm{cm}$ [20]). Furthermore, for $5 \mathrm{~V} N=10^{16} \mathcal{E}_{x}(x)$ is hardly affected by $d$ and is less uniform than for higher $N$. This can be attributed to the very high $\mathcal{E}_{\mathrm{c}, x}(\sim 1.48 \mathrm{MV} / \mathrm{cm})$ compared to $\mathcal{E}_{\mathrm{c}, y}(\sim 0.3 \mathrm{MV} / \mathrm{cm})$. In summary, for low $N$ superjunction RESURF is not needed, but for all other cases for obtaining a constant breakdown field a tenfold lower charge balance in LV SJLEDs is required, which was not reported earlier [16].

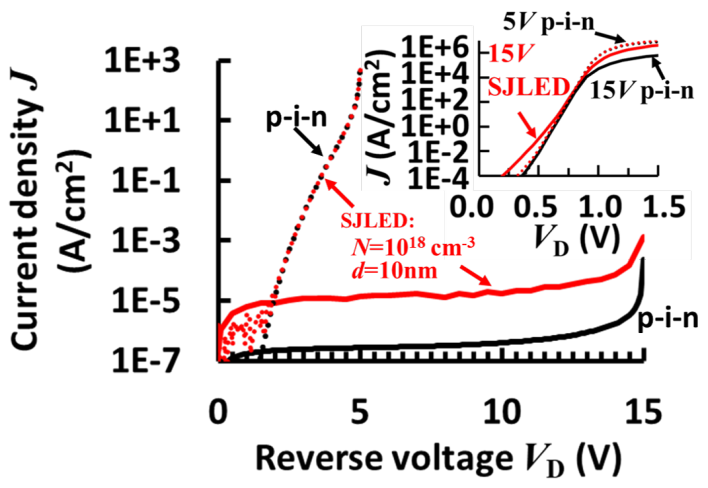

Fig. 5. Reverse $\boldsymbol{I} \boldsymbol{V}$ characteristics of the optimized $5 \mathrm{~V}$ and $15 \mathrm{~V}$ SJLEDs $\left(d=10 \mathrm{~nm}\right.$ for $\left.10^{18} \mathrm{~cm}^{-3}\right)$ compared to those of $5 \mathrm{~V}$ and $15 \mathrm{~V}$ $\mathrm{p}$-i-n diodes (dotted lines). Inset: forward characteristics of the devices.
Further, we study the $I V$ characteristics of the optimized SJLEDs for $N=10^{18} \mathrm{~cm}^{-3}$, see Fig. 5, where default models and parameter values for concentration dependent ShockleyRead Hall (SRH) [25] and Auger recombination, the charge carrier mobility [26] and BTBT [22] are used. The $15 \mathrm{~V}$ devices show lower leakage currents than the $5 \mathrm{~V}$ counterparts due to BTBT. Interestingly, despite the high $N$ in the $5 \mathrm{~V}$ SJLED, BTBT has not increased compared to the $5 \mathrm{~V}$ p-i-n diode due to the RESURF effect. The differences between the characteristics of the 15V SJLED and p-i-n diode at low forward and reverse bias is caused by recombination: the higher doping in the SJLED reduces the (effective) lifetime [25] that in turn increases the leakage and low forward current. In addition, the increased doping in the drift region causes a lower series resistance in the SJLED yielding a higher maximum current density than for the p-i-n diode.

Finally, for $B V \lesssim 5 \mathrm{~V}$ NLA effects will become important in $1 \mathrm{D} \mathrm{p}^{+}$-n diodes [21]. NLA effects can play a role in 1D p-i-n diodes [27], and consequently SJLEDs [28], as well.

It can be derived for the effective field formed by NLA that [29]

$$
\mathcal{E}_{\mathrm{NLA}}(x, y)=\frac{5}{2} \cdot \frac{k \Delta T_{\mathrm{e}}(x, y)}{q \lambda_{\mathrm{e}}},
$$

where $\Delta T_{\mathrm{e}}$ is the increase in electron temperature and $\lambda_{\mathrm{e}}$ is the mean free path of electrons $(\sim 65 \mathrm{~nm}$ in $\mathrm{Si})$. For both the p-i-n diode and SJLED holds [28]

$$
\Delta T_{\mathrm{e}}(x)=\frac{2 q \mathcal{E}_{x}(x) \lambda_{\mathrm{e}}}{5 k} \cdot\left[1-\exp \left(-\frac{x}{\lambda_{\mathrm{e}}}\right)\right] .
$$

For the SJLED an effective vertical field $\left(\mathcal{E}_{\mathrm{NLA}}(y)\right)$ can be obtained similar to that of a single sided junction [29]. Typically, the obtained $\mathcal{E}_{\mathrm{NLA}}(x, y)$ is less than the (local) $\mathcal{E}(x, y)$. For determining the breakdown field Eqs. (3)-(4) should be substituted in the ionization integral that is equated to unity. This basically implies that $B V$ increases for the same device dimensions once NLA becomes important [27].

By adopting Eqs. (3)-(4), Fig. 1(c) shows that particularly for the $5 \mathrm{~V} \mathrm{p}$-i-n diode $\mathcal{E}_{\mathrm{NLA}, x}(x)<\mathcal{E}_{x}(x)\left(\mathcal{E}_{\mathrm{NLA}, x}(x)\right.$ is indicated by the dotted curve), hence NLA is then important. However, it is expected that even in the 15V SJLED, NLA will play a role because of the relatively high vertical ( $y$ direction) field $(\sim 1.2 \mathrm{MV} / \mathrm{cm})$ at $\mathrm{nm}$-scale dimension, even a higher field than for the $5 \mathrm{~V} 1 \mathrm{D} \mathrm{p}^{+}$-n diode. Because $\mathcal{E}_{\mathrm{NLA}, \mathrm{c}, y}$ will then be higher than $\mathcal{E}_{\mathrm{c}, y}$, NLA relaxes the charge balance condition (see Eq. (2)). As a result, especially for $d=10 \mathrm{~nm}$ $N \cdot d$ can then become higher than reported in this work. This requires both a thorough experimental and theoretical future study.

\section{CONCLUSIONS}

An extensive TCAD simulation study has been performed to optimize low-voltage superjunction light-emitting diodes (SJLEDs). The results show that a tenfold more aggressive charge balance condition is required than traditionally reported for power devices. Also, because of the reduced peak field, band-to-band tunneling is less important for SJLEDs than for their conventional counterparts. This work will serve as a guideline for device design in future scaled CMOS nodes. 


\section{REFERENCES}

[1] R. Newman, "Visible light from a silicon p-n junction", Phys. Rev., vol. 100, no. 2, pp. 700-703, 1955.

[2] A.G. Chynoweth and K.G. McKay, "Photon emission from avalanche breakdown in silicon", Phys. Rev., vol. 102, no. 2, pp. 369-376, 1956.

[3] M. D. Plessis, H. Aharoni, and L. W. Snyman, "Silicon LEDs fabricated in standard VLSI technology as components for all silicon monolithic integrated optoelectronic systems", IEEE J. Sel. Topics Quantum Electron., vol. 8, no. 6, pp. 1412-1419, 2002.

[4] B. Huang, X. Zhang, W. Wang, Z. Dong, N. Guan, Z. Zhang, and H. Chen, "CMOS monolithic optoelectronic integrated circuit for on-chip optical interconnection", Optics Communications, vol. 284, no. 16-17, pp. 3924-3927, 2011.

[5] A. Khanmohammadi, R. Enne, M. Hofbauer, and H. Zimmermann, "Monolithically integrated optical random pulse generator in high voltage CMOS technology", Proc. 45th ESSDERC, pp. 138-141, 2015.

[6] V. Agarwal, S. Dutta, A. J. Annema, R. J. E. Hueting, J. Schmitz, M.-J. Lee, E. Charbon and B. Nauta, "Optocoupling in CMOS", International Electron Device Meeting (IEDM), Techn. Digest, pp. 739-742, 2018.

[7] K. Xu, "Silicon MOS Optoelectronic Micro-Nano Structure Based on Reverse-Biased PN Junction", Phys. Stat. Sol., vol. 216, no. 7, p. 1800868, 2019.

[8] S.K. Lazarouk, A.A. Leshok, T.A. Kozlova, A.V. Dolblik, L.D. Vi, V.K. Ilkov, and V.A. Labunov, "3D Silicon Photonic Structures Based on Avalanche LED with Interconnections through Optical Interposer", Int. J. Nanoscience, vol. 18, no. 03n04, p. 1940091, 2019.

[9] M. Krakers, T. Knezevic, K.M Batenburg, X. Liu, and L.K. Nanver, "Diode design for studying material defect distributions with avalanche-mode light emission", IEEE Int. Conf. Microelectronic Test Structures (ICMTS), 2020.

[10] B.P. van Drieënhuizen and R.F. Wolffenbuttel, "Optocoupler based on the avalanche light emission in silicon", Sens. Actuators A: Phys., vol. 31, pp. 229-240, 1992.

[11] L.W. Snyman, M. du Plessis, and H. Aharoni, "Injection-avalanche based $\mathrm{n}^{+} \mathrm{pn}$ silicon complementary metal-oxide-semiconductor light-emitting device $(450-750 \mathrm{~nm})$ with 2-order-of-magnitude increase in light emission intensity", Jpn. J. Appl. Phys., vol. 46, no. 4B, pp. 2474-2480, 2007.

[12] L.W. Snyman, K. Xu, J.-L. Polleux, K.A. Ogudo, and C. Viana, "Higher Intensity SiAvLEDs in an RF Bipolar Process Through Carrier Energy and Carrier Momentum Engineering", IEEE J. Quantum Electr., vol. 51, no. 7, p. 3200110, 2015.

[13] L.W. Snyman, K. Xu, and J. Polleux, "Micron and Nano-Dimensioned Silicon LEDs Emitting at 650 and $750-850 \mathrm{~nm}$ Wavelengths in Standard Si Integrated Circuitry", IEEE J. Quantum Electr., vol. 56, no. 4, p. 3200210, 2020.

[14] S. Dutta, P.G. Steeneken, V. Agarwal, J. Schmitz, A.J. Annema, and R.J.E. Hueting, "The Avalanche-Mode Superjunction LED", IEEE Trans. Electr. Dev., vol. 64, no. 4, pp. 1612-1618, 2017.

[15] R.J.E. Hueting, S. Dutta, V. Agarwal, and A.J. Annema, "Figures of merit of avalanche-mode silicon LEDs", Proc. SPIE, Fifth Conference on Sensors, MEM, and Electro-Optic Systems, p. 1104305, 2019.

[16] J.A. Appels and H.M.J. Vaes, "High voltage thin layer devices (RESURF devices)", International Electron Device Meeting (IEDM), Techn. Digest, pp. 238-241, 1979.

[17] A. Ferrara, B.K. Boksteen, R.J.E. Hueting, A. Heringa, J. Schmitz, and P.G. Steeneken "Ideal RESURF Geometries", IEEE Trans. Electr. Dev., vol. 62, no. 10, pp. 3341-3347, 2015.

[18] F. Udrea, G. Deboy, and T. Fujihira, "Superjunction power devices, history, development, and future prospects", IEEE Trans. Electr. Dev., vol. 64 , no. 3 , pp. 713-727, 2017.

[19] T. Fujihira, "Theory of semiconductor superjunction devices", Jpn. J. Appl. Phys., vol. 36, no. 10, pp. 6254-6262, 1997.

[20] S.M. Sze and K.K. Ng, "Physics of Semiconductor Devices", Hoboken, NJ, USA, Wiley, 3rd edition, 2007.

[21] S. Dutta, R.J.E. Hueting, A.J. Annema, L. Qi, L.K. Nanver, and J. Schmitz, "Opto-electronic modeling of light emission from avalanche-mode silicon p+n junctions", J. Appl. Phys., vol. 118, p. 114506, 2015.

[22] G.A.M. Hurkx, D.B.M. Klaassen, and M.P.G. Knuvers, "A new recombination model for device simulation including tunneling", IEEE Trans. Electr. Dev., vol. 39, no. 2, pp. 331-338, 1992.

[23] Atlas device simulation software, v. 5.30.0.R, Silvaco, Inc., Santa Clara, CA, USA, 2020
[24] S. Selberherr, Analysis and Simulation of Semiconductor Devices, Springer-Verlag, New-York, 1984.

[25] D.J. Roulston, N.D. Arora, and S.G. Chamberlain, "Modeling and Measurement of Minority-Carrier Lifetime versus Doping in Diffused Layers of $\mathrm{n}^{+} \mathrm{p}$ Silicon Diodes", IEEE Trans. Electron Devices, Vol. 29, pp. 284-291, 1982.

[26] D.B.M. Klaassen, "A Unified Mobility Model for Device SimulationI. Model Equations and Concentration Dependence", Solid-State Electronics, vol. 35, no. 7, pp. 953-959, 1992.

[27] P. Agarwal, M. J. Goossens, V. Zieren, E. Aksen, and J. W. Slotboom, "Impact ionization in thin silicon diodes", IEEE Electr. Dev. Lett., vol. 25, no. 12, pp. 807-809, 2004.

[28] S. Dutta, "Avalanche-mode Silicon LEDs for monolithic optical coupling in CMOS technology", PhD thesis, Enschede, November 2017.

[29] J.W. Slotboom, G. Streutker, M.J. van Dort, P.H. Woerlee, A. Pruijmboom, and D.J. Gravesteijn, "Non-local impact ionization in silicon devices", International Electron Device Meeting (IEDM), Techn. Digest, pp. 127-130, 1991.

[30] W. Fulop, "Calculation of avalanche breakdown voltages of silicon p-n junctions", Solid-State Electr., vol. 10, pp. 39-43, 1967. 